Research Article

\title{
Design and Optimum of Dust Collector with Corrugated Plate for Underground Mine
}

\author{
Ming Li $\mathbb{D}^{1},{ }^{1}$ Chao Wu, ${ }^{1}$ Zhi-yong Zhou ${ }^{1},{ }^{1}$ Wei-chun Lian, ${ }^{2}$ and Zhi-xiong Chen ${ }^{2}$ \\ ${ }^{1}$ School of Resources and Safety Engineering, Central South University, Changsha, Hunan, China \\ ${ }^{2}$ Fankou Lead-Zinc Mine, Shenzhen Zhongjin Lingnan Nonfemet Company Limited, Shaoguan, Guangdong, China
}

Correspondence should be addressed to Ming Li; liming_csu@csu.edu.cn

Received 9 June 2018; Revised 5 September 2018; Accepted 24 September 2018; Published 23 October 2018

Guest Editor: Mohsen S. Masoudian

Copyright (C) 2018 Ming Li et al. This is an open access article distributed under the Creative Commons Attribution License, which permits unrestricted use, distribution, and reproduction in any medium, provided the original work is properly cited.

A set of dust collectors was designed with corrugated plate for an underground metal mine, which has low ventilation resistance, simple maintenance, and strong environmental adaptability. A three-dimensional simulation model was built based on ANSYSFluent software, and it was used to analyze the influence law of key parameters on the comprehensive dust removal efficiency; the angle of corrugated plate to the horizontal plane, the surface characteristics of plate, pressure loss and dust removal efficiency were discussed. The optimal design scheme of the dust collector was determined according to the simulation results. The dust collection was carried out in the Fankou lead-zinc underground metal mine in China, and the total dust removal efficiency was more than $95 \%$, and for respiratory dust, it was more than $85 \%$. This dust collector can be widely used in similar underground metal mines.

\section{Introduction}

The mining scale, mining intensity, and mining depth have increased rapidly during recent decades for metal mines in China, especially for old mines with expansion construction and reconstruction to improve its mining capacity. Consequently, it makes the ventilation network complex and the structure irrational, ventilation circuit long, ventilation resistance big, and daily management much difficult, and it brings serious contradiction between air supply and demand. At present, the existing well and roadways are used as the inlet shaft or airway, which is one of the important ways to effectively solve the poor ventilation problem for underground mines. Some metal mines allow fresh air inlet from the main shaft to partially replenish the demand of air under certain conditions. Due to the small ventilation resistance and large cross section, the volume of airflow is large, and the fresh air is polluted by dust in the skip shaft during the real application. According to the provisions from Safety Regulations of Metal and Nonmetal Mines (GB16423-2006), "The skip shaft should not double as intake shaft. When mixture shaft is used as the intake shaft, it should take effective purification measures to ensure the quality of the air source." So these metal mines face the problem of purifying airflow which is from the main shaft or skip shaft.

Dust collectors have been widely used because of technology ripening, safety working, and economy reasonable nowadays. There are many kinds of dedusting technologies and dust collectors [1], most of which focus on wet dedusting and filter removal technologies, especially on spraying dedusting [2-4] and filter-bag dedusting. Although current dedusting and dust collectors are relatively mature technology, it is not easy to apply them well into underground mines, which needs to meet the conditions of confined space, easy maintenance, efficiency, and the large volume of airflow economically [5]. It is of great practical significance and application value to investigate the purification technology and dedusting device for underground metal mine.

\section{Design of Dust Collector}

In order to meet the requirements for the purification of air in underground metal mine (e.g., low ventilation resistance, high capacity to deal with airflow, and relatively low content of respiration dust), the dedusting types of shutter, spray, and water film need to be combined in the design of the dust collector. The corrugated plate was selected as the main 


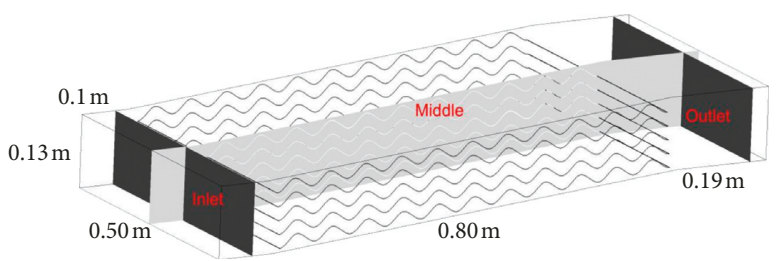

(a)

\section{Velocity}

Volume rendering 1

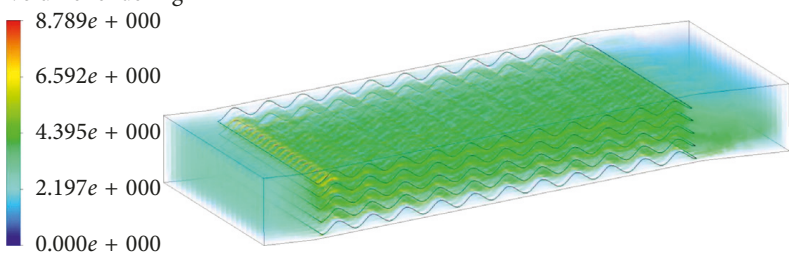

$\left(\mathrm{m} \mathrm{s}^{-1}\right)$

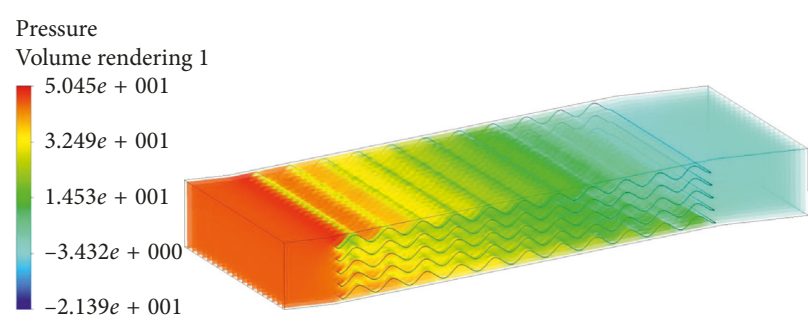

$(\mathrm{Pa})$

(b)

Particle mass concentration

Contour 1

$3.782 e-002$

$3.404 e-002$
$3.025 e-002$

$3.025 e-002$
$2.647 e-002$

$2.647 e-002$
$2.269 e-002$

$2.269 e-002$
$1.891 e-002$

$1.891 e-002$

$1.513 e-002$
$1.135 e-002$

$1.135 e-002$
$7.564 e-003$
$3.782 e-003$

$3.782 e-003$

$0.000 e+000$

$\left(\mathrm{kg} \mathrm{m}^{-3}\right)$

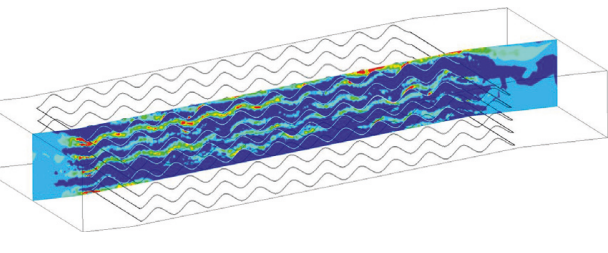

(d)

(c)

FIgURE 1: Simulating graphs from ANSYS-Fluent. (a) Geometry. (b) Pressure. (c) Velocity. (d) Particle mass concentration.

material, and the idea of modular design was adopted to build the dust collector. One or more sets of independent dust removal module were used to constitute the dust collector, and these modules can be used in series, parallel, and series-parallel modes.

2.1. Preliminary Design. Each dedusting module consists of a metal support and corrugated plate. The corrugated plate is fixed on the metal support and has an angle to the horizontal plane and formed like a shutter-type dust collector [6]. When the dusty airflow passes through the corrugated plate, the airflow disturbance caused by the wave-shaped structure of the corrugated plate would enhance the inertia effect and increase the collision probability between the dust and the surface of the corrugated plate [7]. The dust removal mechanism of the corrugated plate includes gravity sedimentation, inertia separation, surface adhesion $[8,9]$, and so on.

2.2. Simulation and Analysis. The technology of numerical simulation is widely used to assist experimental research and provides more and faster simulation data $[10,11]$. A numerical model was built to optimize the parameters of the dedusting module. The dedusting efficiency, installation angle, ventilation resistance, pressure loss, and flow-field distribution were simulated and discussed. The numerical model used in this study is implemented by solving the differential equations of conservation of mass, momentum, and energy and is complemented by turbulent flow models. Calculations are performed with the commercial software package ANSYS-Fluent. The discrete phase model (DPM) is used to simulate the results of dust [12]. The trajectory of a discrete phase particle is simulated by integrating the force balance on the particle in a Lagrangian reference frame. This force balance equates the particle inertia with the forces acting on the particle [13] and can be written (for the $x$ direction in Cartesian coordinates) as follows:

$$
\left\{\begin{array}{l}
\frac{d u_{p}}{d t}=F_{\mathrm{D}}\left(\vec{u}-\vec{u}_{p}\right)+\frac{\vec{g}\left(\rho_{p}-\rho\right)}{\rho_{p}}+\vec{F}, \\
F_{\mathrm{D}}=\frac{18 \mu}{\rho_{p} d_{p}^{2}} \frac{C_{\mathrm{D}} \operatorname{Re}}{24}, \\
\operatorname{Re} \equiv \frac{\rho d_{p}\left|\vec{u}_{p}-\vec{u}\right|}{\mu},
\end{array}\right.
$$

where $\vec{F}$ is an additional acceleration (force/unit particle mass) term, $F_{\mathrm{D}}\left(\vec{u}-\vec{u}_{p}\right)$ is the drag force per unit particle mass, $\vec{u}$ is the fluid phase velocity, $\vec{u}_{p}$ is the particle velocity, $\mu$ is the molecular viscosity of the fluid, $\rho$ is the fluid density, $\rho_{p}$ is the density of the particle, $d_{p}$ is the particle diameter, and Re is the relative Reynolds number.

The simulation geometry is shown in Figure 1(a). The computational domain was $1.1 \mathrm{~m} \times 0.5 \mathrm{~m} \times 0.13 \mathrm{~m}$ and included five corrugated plates. Three planes (inlet, outlet, and middle) were selected to calculate the simulation results. The three angles $\left(0^{\circ}, 5^{\circ}\right.$, and $\left.10^{\circ}\right)$ between the horizontal plane to the corrugated plate and the surface of the corrugated plate were set as three types, i.e., "two sides" means that the boundary condition is "trap" for both surfaces of the corrugated plate, "one side" represents that upper surface of the corrugated plate was set as "trap," and "no side" refers to that no surface was set as "trap." Typical simulation results and graphs are shown in Figures 1(b)-1(d).

Three planes were selected to evaluate the dust removal efficiency, as shown in Figure 1(a). The data were averaged value of three planes (inlet, outlet, and middle) according to the simulation results, which are shown in Figures 2 and 3. 


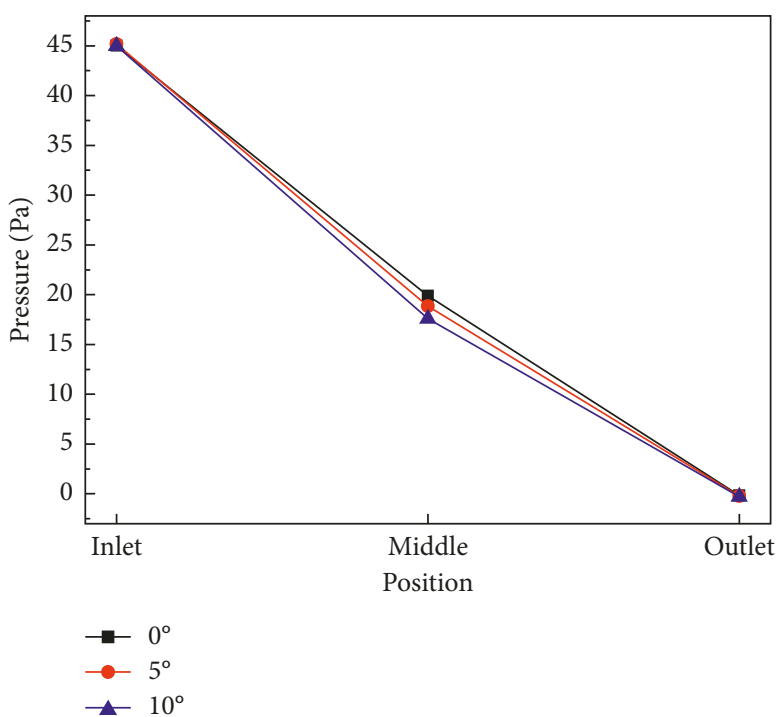

(a)

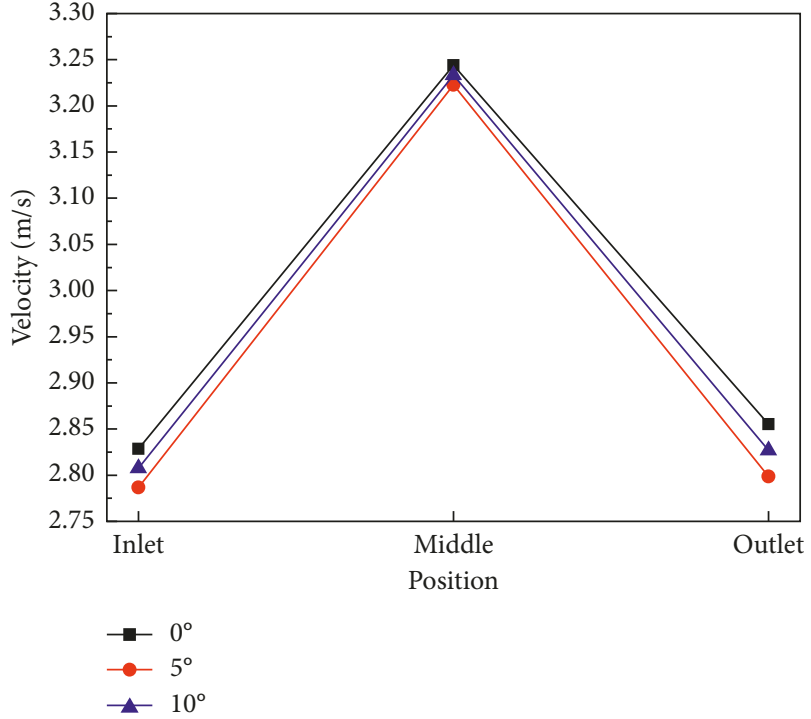

(b)

Figure 2: Variation of (a) pressure and (b) velocity for dust collector.

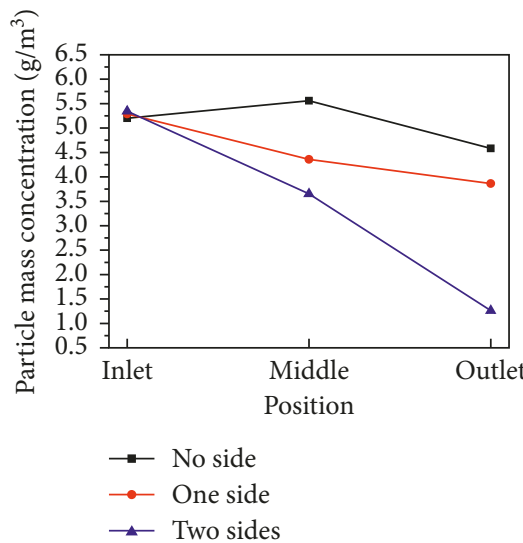

(a)

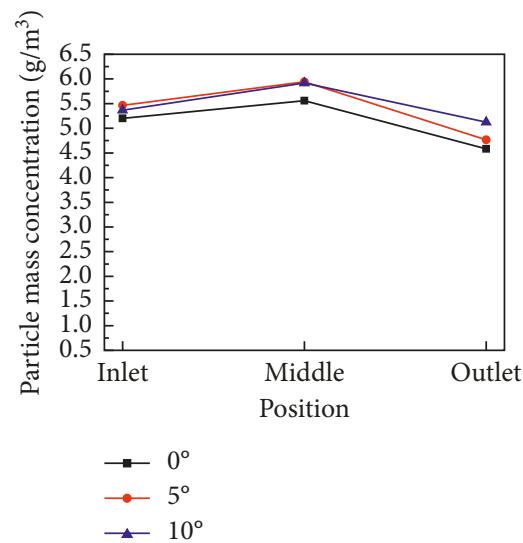

(d)

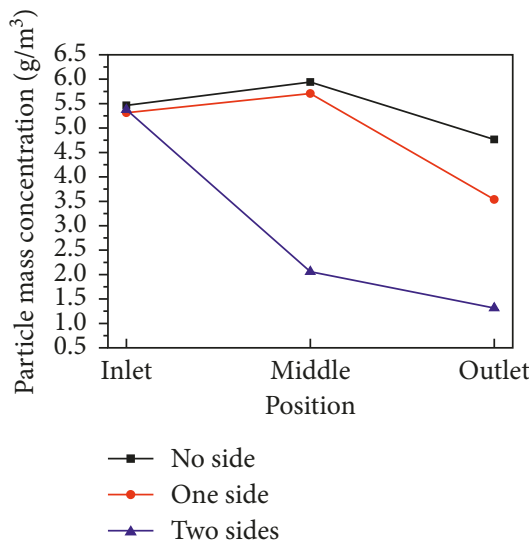

(b)

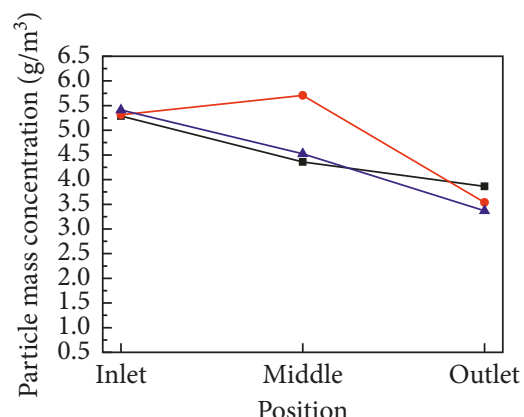

$\rightarrow 0^{\circ}$
$\rightarrow 5^{\circ}$
$\longleftarrow 10^{\circ}$

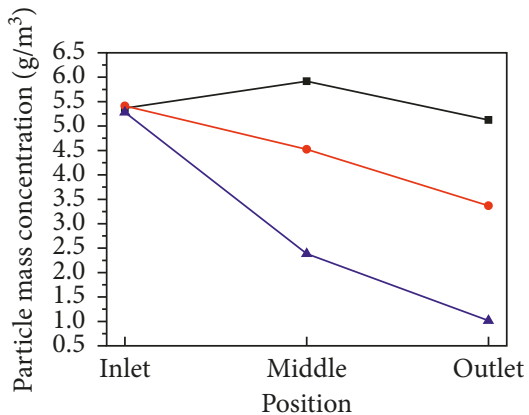

- No side

$\rightarrow$ One side

$\triangle$ Two sides

(c)

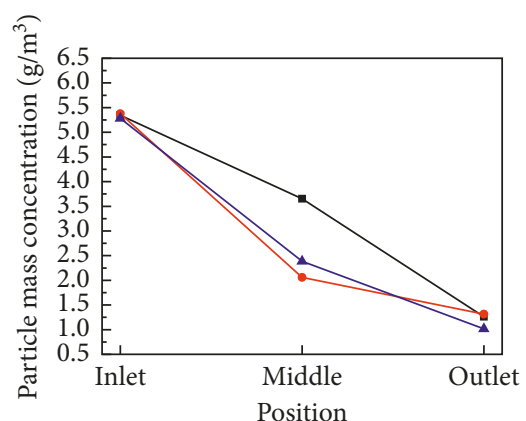

$\rightarrow-0^{\circ}$

$\rightarrow 10^{\circ}$

(e)

(f)

Figure 3: Simulation results of dedusting effect for the corrugated plate. (a) Angle is $0^{\circ}$. (b) Angle is $5^{\circ}$. (c) Angle is $10^{\circ}$. (d) No side trap for dust. (e) One side trap for dust. (f) Two sides trap for dust. 


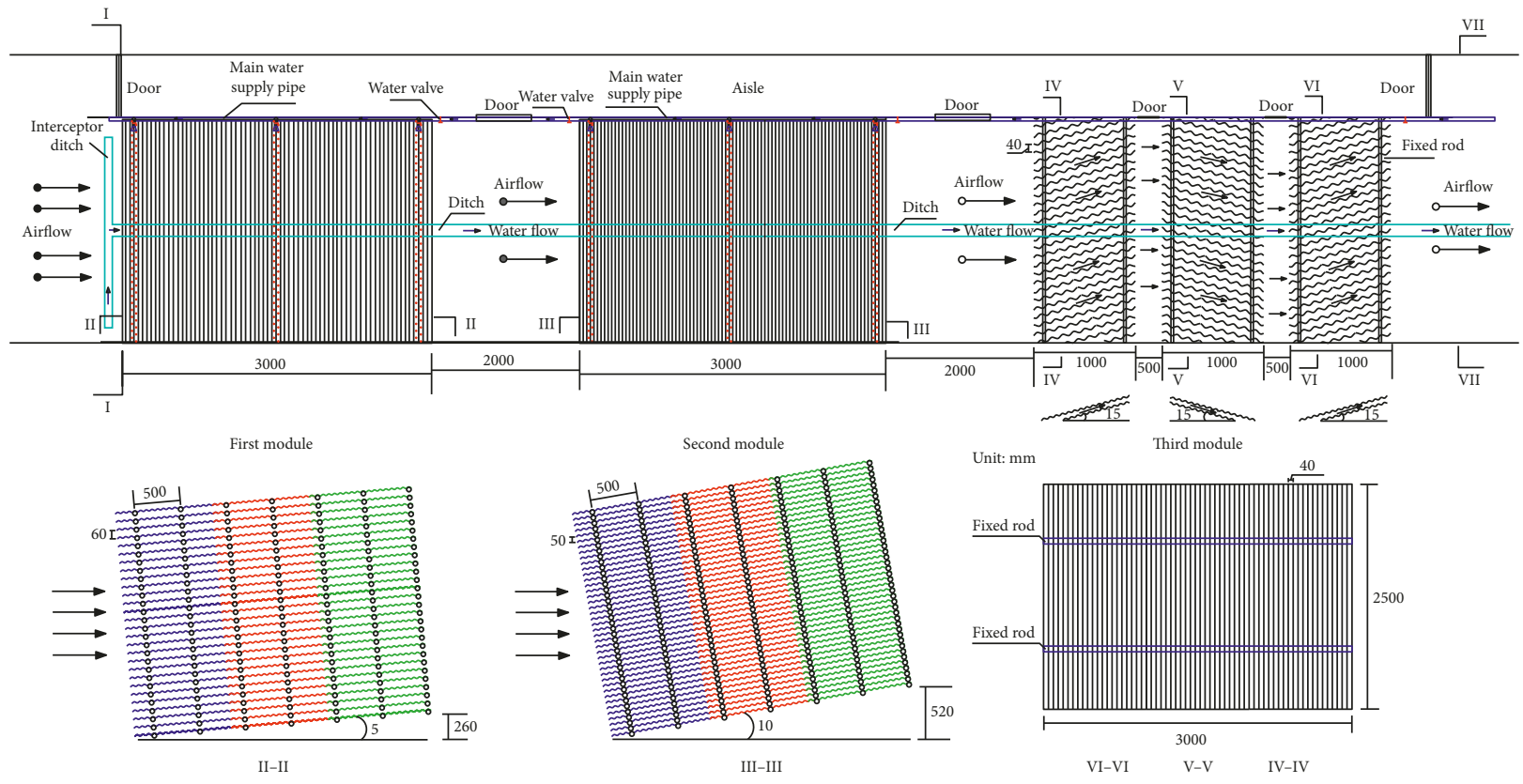

Figure 4: Design diagram of the dust collector for underground mine.
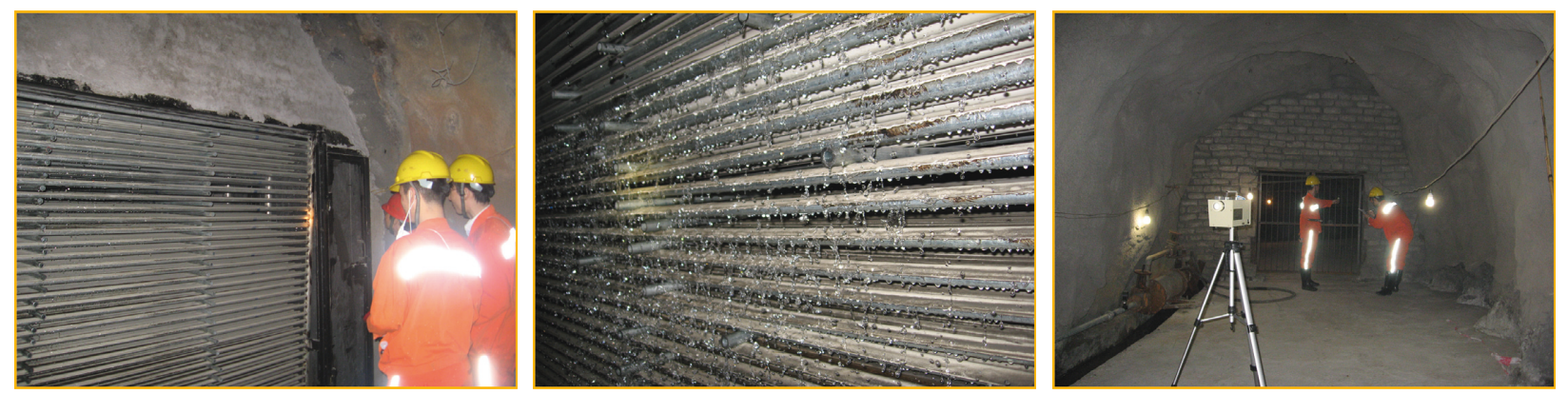

Figure 5: Photos of dust collector in underground metal mine.

Figure 2(a) shows the pressure loss from inlet plane to outlet plane is about $45 \mathrm{~Pa}$, and the installation angle has little effect on the pressure loss. The simulation velocity was set as $2-3 \mathrm{~m} / \mathrm{s}$ in order to meet the processing ability of the volume of airflow in Figure 2(b).

Figures 3(a)-3(c) show that the dust removal efficiency is dependent on the plate area and the characteristics of the plate surface, and the dedusting efficiencies were as follows: two sides $>$ one side $>$ no side. The collection efficiency is $76.38 \%$ for two sides, $26.97 \%$ for one side, and $11.83 \%$ for no side at angle $0^{\circ} ; 75.55 \%$ for two sides, $34.05 \%$ for one side, and $12.79 \%$ for no side at angle $5^{\circ} ; 80.75 \%$ for two sides, $37.39 \%$ for one side, and $4.6 \%$ for no side at angle $10^{\circ}$.

Figures 3(d)-3(f) present that the dust removal efficiency is affected by the angle, but its effect is not so significant, and it depends on the surface area of plate, which is associated with the "trap" type boundary condition [14].

In order to improve the dust capture capability for the surface of the corrugated plate and to increase the comprehensive efficiency of dust removal, the water supply pipe was installed on one or both sides of each dedusting module, with water sprinkled into its inside through injection hole.
Some leakage holes were arranged on each corrugated plate, and water curtain was formed between the plates through these leakage holes. Water curtain became the water mist when it was flapped and impacted by airflow, and water film was formed on the surface of the corrugated plate. The inertial effect, wet dedusting, and water film collecting constituted the dust collector. Because of the spoiler effect of the corrugated plate and spray dust removal system, the dust removal rate is high for respiratory dust. The dust was taken away by sprinkle water, which prevented dust aggregation on the surface and washed the dust.

3. Field Application and Effect Evaluation. The dust collector has been applied in a large nonferrous metal mine to purify airflow from the main shaft, and the device was installed in a roadway with a size of $25.0 \mathrm{~m} \times 4.5 \mathrm{~m} \times 4.0 \mathrm{~m}$. The dust collector consists of three independent dedusting modules, and the basic structure is shown in Figure 4.

Field application results: the dust concentration is 5$10 \mathrm{mg} / \mathrm{m}^{3}$ for the inlet airflow from the main shaft and $0.5-1.5 \mathrm{mg} / \mathrm{m}^{3}$ for the outlet airflow from this dust collector; 
the concentration of respiratory dust is $0.15-0.4 \mathrm{mg} / \mathrm{m}^{3}$ falling below the standard (the concentration of respiratory dust $\leq 0.5 \mathrm{mg} / \mathrm{m}^{3}$ ) for inlet fresh air; the throughput volume of this dust collector is $15-20 \mathrm{~m}^{3} / \mathrm{s}$; the ventilation resistance is $200-300 \mathrm{~Pa}$; the volume of sprinkle water is $200-300 \mathrm{~m}^{3} / \mathrm{d}$; and the photos of this dust collector are shown in Figure 5. The adoption of the modular design in this dust collector provides the capability to change the parameter according to field requirements. Field application proved that the dust collector meets the design requirements and can be used into the underground metal mine for the purification of the inlet air from the main shaft or skip shaft.

Based on the ventilation cost of this applied mine, the main economic indicator is that the operation cost of ventilation is about RMB30,000 per year for $1 \mathrm{~m}^{3}$ fresh air from auxiliary shaft. According to these data, the throughput volume of this dust collector is $20 \mathrm{~m}^{3}$; therefore, the annual economic benefit of RMB450,000 can be achieved.

\section{Conclusions}

This study was conducted to design a new dust collector to purify airflow for the main shaft in an underground metal mine. The corrugated plate was selected as the main material; ANSYS-Fluent software was used to optimize the parameter of the dust collector. The modular design was used in the dust collector, which provides the capability to change module composition according to field requirements. The dust collector integrates wet dedusting, condensation, collision, inertia, gravity, and other dedusting methods.

This device was applied in an underground metal mine, which has low ventilation resistance, simple maintenance, and strong environmental adaptability. Field application proved that the dust collector meets the design requirements and can be used into the underground metal mine for the purification of the inlet air from the main shaft or skip shaft.

\section{Data Availability}

The data used to support the findings of this study are available from the corresponding author upon request.

\section{Conflicts of Interest}

The authors declare that they have no conflicts of interest.

\section{Acknowledgments}

This research was supported by the National Natural Science Foundation of China (No. 51674289) and National Key Research and Development Plan of China (Nos. 2017YFC080520405 and 2018YFC0808404).

\section{References}

[1] D. Zhang and C. Wang, Manual of Dust Collector, Chemical Industry Press, Beijing, China, 2nd edition, 2015.

[2] M. W. Tessum and P. C. Raynor, "Effects of spray surfactant and particle charge on respirable coal dust capture," Safety and Health at Work, vol. 8, no. 3, pp. 296-305, 2017.
[3] H. Wang, C. Wang, and D. Wang, "The influence of forced ventilation airflow on water spray for dust suppression on heading face in underground coal mine," Powder Technology, vol. 320, pp. 498-510, 2017.

[4] G. Zhou, Q. Zhang, R. Bai, T. Fan, and G. Wang, “The diffusion behavior law of respirable dust at fully mechanized caving face in coal mine: CFD numerical simulation and engineering application," Process Safety and Environmental Protection, vol. 106, pp. 117-128, 2017.

[5] J. Csavina, J. Field, M. P. Taylor et al., "A review on the importance of metals and metalloids in atmospheric dust and aerosol from mining operations," Science of the Total Environment, vol. 433, pp. 58-73, 2012.

[6] J. B. Sim, U. H. Yeo, G. H. Jung, S. B. Park, G. N. Bae, and S. J. Yook, "Enhancement of louver dust collector efficiency using modified dust container," Powder Technology, vol. 325, pp. 69-77, 2018.

[7] K. Vohra, K. Ghosh, S. N. Tripathi et al., "Submicron particle dynamics for different surfaces under quiescent and turbulent conditions," Atmospheric Environment, vol. 152, pp. 330-344, 2017.

[8] C. Wu and L. ming, Particle Adhesion and Removal, Metallurgical Industry Press, Beijing, China, 2014.

[9] L. ming, C. Wu, Y. j. Liu, and Y. hui, "Effect of reagent films on the coupling relationship between glass surface and particles," Transactions of Nonferrous Metals Society of China, vol. 22, pp. 2799-2805, 2012.

[10] L. Liu, Z. Y. Fang, Y. P. Wu, X. P. Lai, P. Wang, and K. L. Song, "Experimental investigation of solid-liquid two-phase flow in cemented rock-tailings backfill using electrical resistance tomography," Construction and Building Materials, vol. 175, pp. 267-276, 2018.

[11] W. Nie, W. Wei, P. Cai et al., "Simulation experiments on the controllability of dust diffusion by means of multi-radial vortex airflow," Advanced Powder Technology, vol. 25, no. 3, pp. 835-847, 2018.

[12] L. ming, S. M. Aminossadati, and W. chao, "Numerical simulation of air ventilation in super-large underground developments," Tunnelling and Underground Space Technology, vol. 52, pp. 38-43, 2016.

[13] T. Ren, Z. Wang, and G. Cooper, "CFD modelling of ventilation and dust flow behaviour above an underground bin and the design of an innovative dust mitigation system," Tunnelling and Underground Space Technology, vol. 41, pp. 241-254, 2014.

[14] L. ming, C. Wu, and W. Pan, "Sedimentation behavior of indoor airbome microparticles," Journal of China University Mining and Technology, vol. 18, no. 4, pp. 588-593, 2008. 


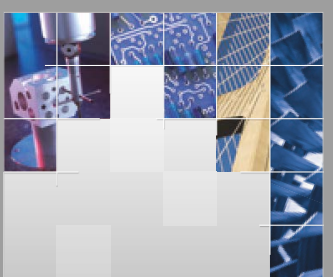

\section{Enfincering}
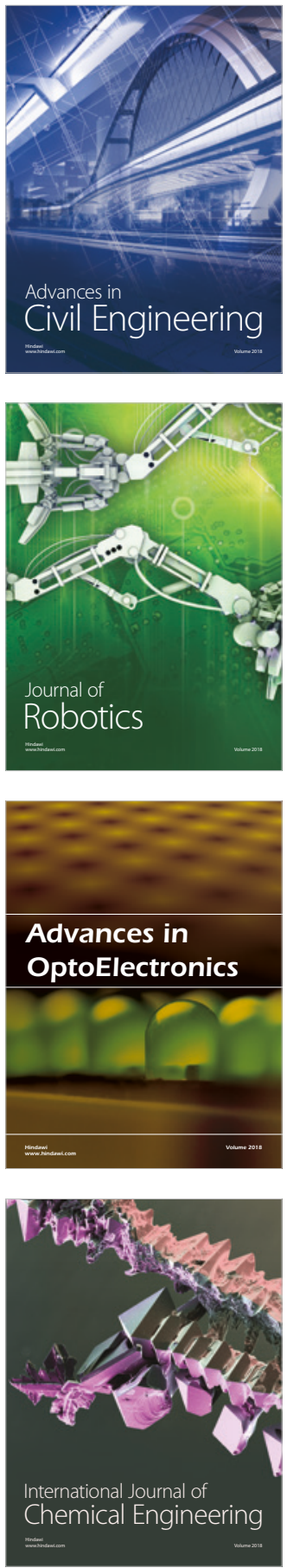

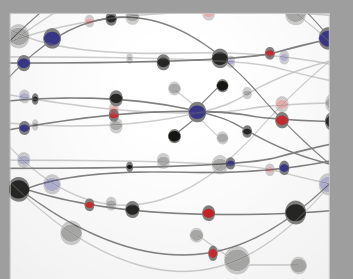

\section{Rotating \\ Machinery}

The Scientific World Journal

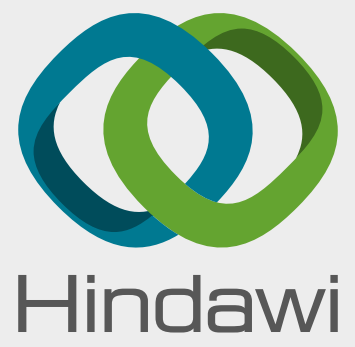

Submit your manuscripts at

www.hindawi.com
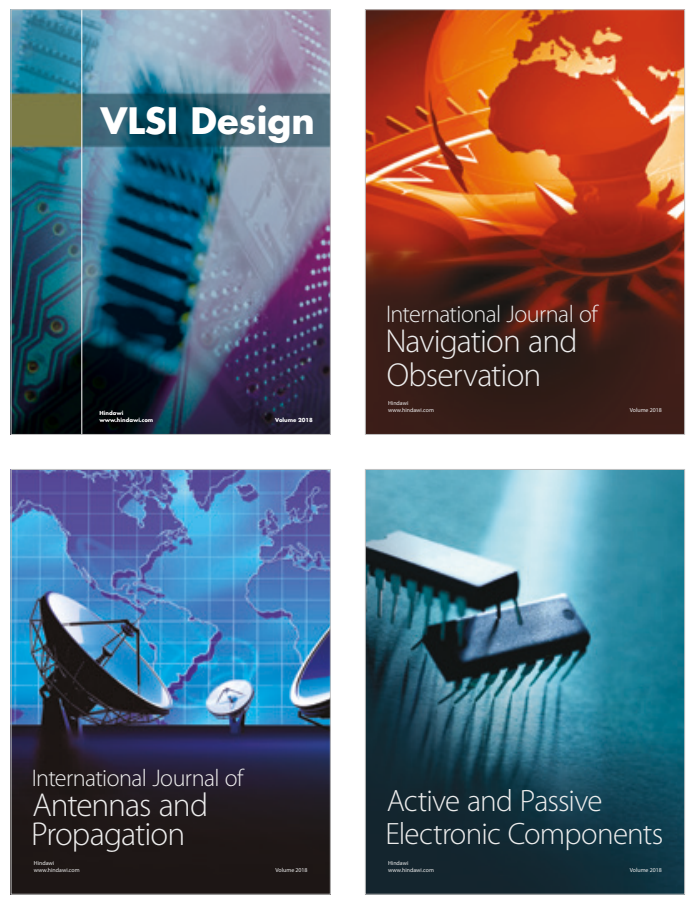
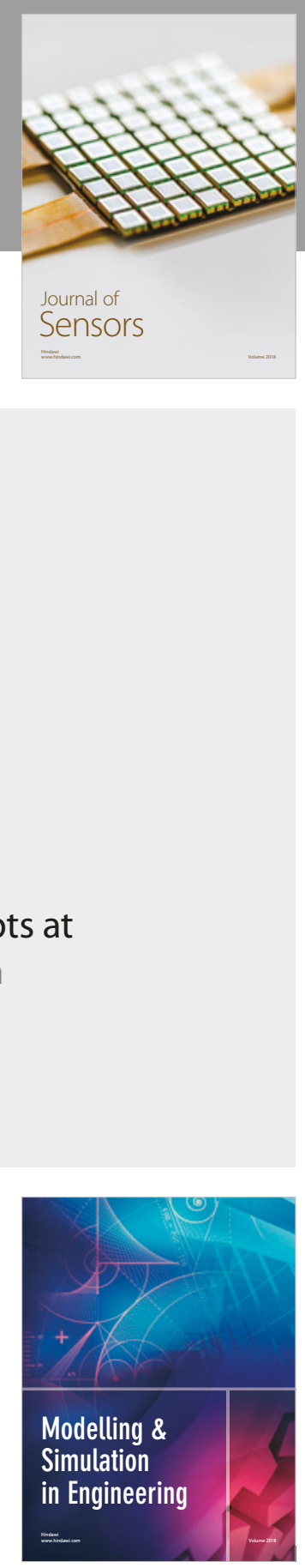

\section{Advances \\ Multimedia}
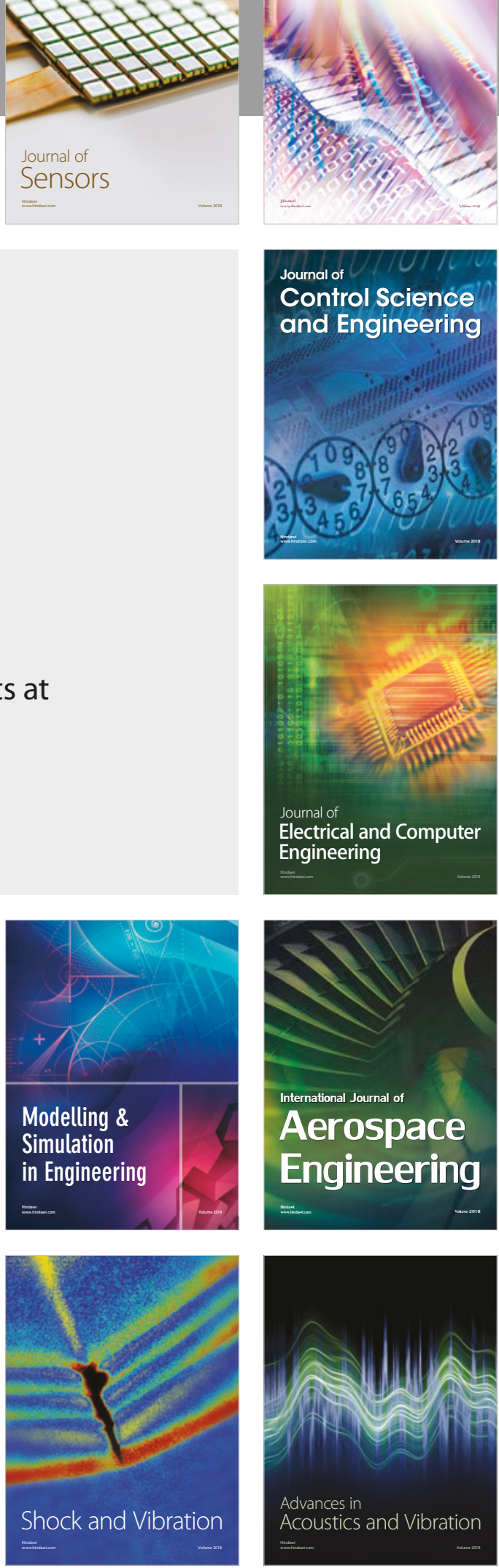